\title{
(6) OPEN ACCESS \\ Cerebral glucose metabolism and cognition in newly diagnosed Parkinson's disease: ICICLE-PD study
}

\author{
M J Firbank, ${ }_{1}^{1}$ A J Yarnall, ${ }^{1}$ R A Lawson, ${ }^{1} \mathrm{G}$ W Duncan, ${ }^{2} \mathrm{~T} \mathrm{~K} \mathrm{Khoo,}^{3} \mathrm{G}$ S Petrides, ${ }^{4}$ \\ J T O'Brien, ${ }^{5}$ R A Barker, ${ }^{6}$ R J Maxwell, ${ }_{1}^{7}$ D J Brooks, ${ }^{1,8,9}$ D J Burn ${ }^{1}$
}

- Additional material is published online only. To view please visit the journal online (http://dx.doi.org/10.1136/ jnnp-2016-313918).

For numbered affiliations see end of article.

\section{Correspondence to} Dr Michael Firbank, Institute of Neuroscience and Newcastle University Institute for Ageing, Newcastle University, Newcastle on Tyne NE4 5PL, UK; michael. firbank@ncl.ac.uk

Received 3 May 2016 Revised 14 July 2016 Accepted 19 August 2016 Published Online First 6 October 2016

\section{CrossMark}

To cite: Firbank MJ, Yarnall AJ, Lawson RA, et al. J Neurol Neurosurg Psychiatry 2017:88:310-316.

\section{ABSTRACT}

Objective To assess reductions of cerebral glucose metabolism in Parkinson's disease (PD) with 18Ffluorodeoxyglucose (FDG) positron emission tomography (PET), and their associations with cognitive decline.

Methods FDG-PET was performed on a cohort of 79 patients with newly diagnosed PD (mean disease duration 8 months) and 20 unrelated controls. PD participants were scanned while on their usual dopaminergic medication. Cognitive testing was performed at baseline, and after 18 months using the Cognitive Drug Research (CDR) and Cambridge Neuropsychological Test Automated Battery (CANTAB) computerised batteries, the Mini-Mental State Examination (MMSE), and the Montreal Cognitive Assessment (MoCA). We used statistical parametric mapping (SPM V.12) software to compare groups and investigate voxelwise correlations between FDG metabolism and cognitive score at baseline. Linear regression was used to evaluate how levels of cortical FDG metabolism were predictive of subsequent cognitive decline rated with the MMSE and MoCA.

Results PD participants showed reduced glucose metabolism in the occipital and inferior parietal lobes relative to controls. Low performance on memory-based tasks was associated with reduced FDG metabolism in posterior parietal and temporal regions, while attentional performance was associated with more frontal deficits. Baseline parietal to cerebellum FDG metabolism ratios predicted MMSE ( $\beta=0.38, p=0.001)$ and MoCA ( $\beta=0.3$, $p=0.002$ ) at 18 months controlling for baseline score. Conclusions Reductions in cortical FDG metabolism were present in newly diagnosed PD, and correlated with performance on neuropsychological tests. A reduced baseline parietal metabolism is associated with risk of cognitive decline and may represent a potential biomarker for this state and the development of PD dementia.

\section{INTRODUCTION}

Parkinson's disease (PD) is a progressive neurodegenerative disorder affecting over four million people above the age of 50, with the prevalence expected to double to 9.3 million by $2030 .{ }^{1}$ There has been a gradual shift in the definition of PD, from a primary movement disorder to a multisystem neurodegenerative condition affecting multiple cognitive domains. Over $40 \%$ of early PD cases are affected by mild cognitive impairment (PD-MCI) ${ }^{2}$ and up to $80 \%$ of patients with PD will eventually develop dementia (PDD). ${ }^{3}$
The neural substrates of cognitive impairment in PD remain poorly understood and there is a need to establish imaging biomarkers which could aid in the identification of patients at risk of cognitive decline, for early diagnosis and for therapeutic trials.

A number of studies have established associations between low cognitive ratings in prevalent PD and regional reductions in cortical glucose metabolism, mostly involving posterior regions. ${ }^{4} 5$ However, studies investigating the power of $18 \mathrm{~F}$-fluorodeoxyglucose (FDG) positron emission tomography (PET) to predict later cognitive decline have been fewer, and chiefly performed in small samples with relatively advanced PD. ${ }^{6-8}$

The inferior parietal lobe is well established as a region which shows reduced glucose metabolism and perfusion in a range of dementias, including Alzheimer's disease, dementia with Lewy bodies (DLB) and PDD. Reduced parietal metabolism is apparent at the early MCI stage. ${ }^{5-11}$ We hypothesised that reduced FDG metabolism in this region would be predictive of cognitive decline in early PD.

In the present study, our aims were to: (1) establish the pattern of glucose metabolism in early stage PD relative to healthy controls; (2) investigate the association between performance on specific cognitive tests and levels of regional glucose metabolism; (3) to evaluate whether parietal metabolism predicted cognitive decline over 18 months.

\section{METHODS}

\section{Participants}

Participants were taking part in the Incidence of Cognitive Impairment in Cohorts with Longitudinal Evaluation-Parkinson's Disease (ICICLE-PD) study. ${ }^{2}$ They were newly diagnosed PD $(n=158)$ from community and outpatient clinics in the North East of England or unrelated healthy controls $(n=99)$. PD was diagnosed by a movement disorders specialist according to the UK Brain Bank criteria ${ }^{12}$ and a repeat clinical evaluation was performed at follow-up to increase diagnostic accuracy. The first $81 \mathrm{PD}$ and 20 controls who consented to scanning received a FDG-PET scan. Of the $81 \mathrm{PD}$ participants scanned, 2 were excluded due to change of diagnosis at follow-up. Thus, 79 PD participants and 20 healthy controls who consented to a FDG-PET scan were included in this study. The PD participants had their PET scan at baseline, while the controls were scanned 
$\sim 3$ years after baseline. Full inclusion and exclusion criteria have been previously described. ${ }^{2}$

\section{Standard protocol approvals, registrations and patient} consents

The study was approved by the Newcastle and North Tyneside Research Ethics Committee and performed according to the Declaration of Helsinki. All participants provided written informed consent.

\section{Clinical assessment}

Clinical assessments were performed by trained examiners, and included a standardised neurological examination, with rating of motor symptoms using the revised Movement Disorders Society-Unified Parkinson's Disease Rating Scale (MDS-UPDRS III) ${ }^{13}$ and Hoehn and Yahr $^{14}(\mathrm{H} \& Y)$ staging.

\section{Neuropsychological assessment}

Global cognitive function was assessed using the Mini-Mental State Examination (MMSE) ${ }^{15}$ and the Montreal Cognitive Assessment (MoCA). ${ }^{16}$ In accordance with MDS Task Force recommendations, ${ }^{17}$ five cognitive domains were assessed. Attention was measured using the Cognitive Drug Research computerised battery. ${ }^{18}$ Mean response times of simple reaction time, choice reaction time and digit vigilance were summed to produce a Power of Attention score. Digit vigilance accuracy was also evaluated as part of this domain. Power of Attention (PoA) and Digit Vigilance (DV) data were missing at baseline on one PD participant. Memory was assessed with Pattern Recognition Memory (PRM), Spatial Recognition Memory (SRM), and Paired Associates Learning (PAL) from the computerised Cambridge Neuropsychological Test Automated Battery (CANTAB). ${ }^{19}$ Baseline CANTAB data were not acquired on two PD participants. Executive function was determined using the modified One Touch Stockings (OTS) version of the Tower of London task from CANTAB, phonemic fluency (words beginning with $\mathrm{F}, \mathrm{A}$ and $\mathrm{S}$ in $1 \mathrm{~min}$ each) and semantic fluency (animals in $90 \mathrm{~s}$ ). Fluency was not performed at baseline on one PD participant. The pentagon copying item of the MMSE was graded using a modified 0-2 rating scale as a measure of visuospatial function. Language domain was assessed using the naming (0-3) and sentence (0-2) subsets of the MoCA test. All participants were assessed in an 'on' state, taking their usual dopaminergic medication at baseline and again after 18 months. Levodopa equivalent daily dose (LEDD) value was calculated using the Tomlinson et $a l^{20}$ formula. Baseline MoCA was not performed on the first seven PD participants. Assessments were repeated at 18 months on all the controls, and 90\% (71/79) of the PD participants. Reasons for attrition included loss to follow-up $(n=4)$, declined follow-up $(n=2)$ and death $(n=2)$.

Participants were diagnosed as PD-MCI if they were impaired (1.5 SDs) below normative means (derived from controls) on two or more cognitive tests. ${ }^{2}$ Consistent with previous methodology $^{2}$ modified level 2 criteria were used, as our neuropsychological battery predated the publication of the PD-MCI criteria, with only one test (ie, pentagon copying) being specific to the visuospatial domain.

\section{PET image acquisition}

FDG-PET was acquired over $10 \mathrm{~min}$ at Newcastle University using a Siemens Biograph 40 Truepoint PET-CT starting $30 \mathrm{~min}$ after intravenous administration of $250 \mathrm{MBq}$ F-18 FDG. Siemens software was used for iterative reconstruction (OSEM2D 6 iterations, 16 subsets) with model-based scatter correction and attenuation correction based on the CT scan data obtained immediately before the FDG-PET scan. Participants were asked to fast for 4 hours preinjection and blood glucose was tested to ensure it was $<180 \mathrm{mg} / \mathrm{dL}$. Participants were injected in quiet surroundings with minimal distractions and with their eyes open. The patients with PD were scanned in an 'on' state, after taking their usual dopaminergic medication.

\section{PET image processing}

Images of FDG metabolism were processed using SPM V.12 software (http://www.fil.ion.ucl.ac.uk/spm/). All images were spatially normalised to an age appropriate FDG-PET template in Montreal Neurological Institute (MNI) space, ${ }^{21}$ resampled at $2 \times 2 \times 2 \mathrm{~mm}$, and smoothed with a $8 \mathrm{~mm}$ full width half maximum Gaussian kernel. Voxelwise statistics were performed with the general linear model in SPM V.12 with image intensity proportionally scaled to the whole brain mean for each participant, and using a brain mask of 0.8 mean intensity. Statistics are shown thresholded either after a voxelwise family wise error (FWE) correction for multiple comparisons at $\mathrm{p}<0.05$, or with an uncorrected voxelwise threshold of $\mathrm{p}<0.001$ followed by $\mathrm{p}<0.05$ FWE clusterwise threshold. Group differences were performed with age and sex as covariates. Correlation within the PD group between FDG and cognitive ratings had age, sex and years of education as covariates.

In our recent FDG study on Alzheimer's and Lewy body dementias, ${ }^{9}$ we found that the ratio of FDG in angular gyrus/ cerebellum was significantly reduced in these groups. We therefore investigated the power of this ratio to predict cognitive decline in PD.

\section{Statistical analysis}

Statistical analyses were performed using SPSS V.22.0 (IBM, New York, USA). Means were compared using unpaired t-tests or analysis of variance. The $\chi^{2}$ test was used to compare categorical variables. Change in cognitive and motor score between baseline and repeat was assessed with paired t-tests. Linear regression (continuous dependent variables) was used to investigate the relationship between FDG angular gyrus/cerebellum ratio and cognitive score (MMSE and MoCA) at 18 months with baseline score as covariate. Model fit was checked by examining the residuals.

\section{RESULTS}

Participant demographics are shown in table 1. PD and control groups were well matched for age and sex. There were no significant differences between PD with and without MCI at baseline in disease duration or use of dopaminergic medication, although the PD-MCI participants had a greater motor severity and fewer years of education.

Within the PD group, 41 participants were classified as PD-MCI at baseline while the remaining 38 PD participants were classified as having normal cognitive function (PD-NC). The PD-MCI group, as expected, performed significantly worse on all neuropsychological assessments than PD-NC and control participants (see online supplementary table e-1). There were no significant differences in cognition between the control and PD-NC group in any test, with the exception of SRM, in which the PD-NC performed marginally worse than controls.

Compared with the control participants, both the cognitively normal PD and the PD-MCI groups had reduced FDG metabolism in the occipital lobe (figure 1). The PD-MCI group, in addition, had reduced metabolism in the inferior parietal and posterior temporal regions. There were, however, no significant 
Table 1 Participant demographics

\begin{tabular}{|c|c|c|c|c|}
\hline & Control $(\mathrm{N}=20)$ & PD-NC (N=38) & $\mathrm{PD}-\mathrm{MCl}(\mathrm{N}=41)$ & \\
\hline Age in years at PET scan [range] & $71.9(9.7)[54-91]$ & $72.3(6.4)[57-88]$ & $74.2(4.8)[66-86]$ & $F_{2,96}=1.2, \mathrm{p}=0.31$ \\
\hline Sex M:F & $12: 8$ & $27: 11$ & $30: 42$ & $\chi^{2}=0.95, p=0.6$ \\
\hline Years education & $13.9(3.8)$ & $13.3(3.6)$ & $10.9(3.3)$ & $F_{2,96}=6.8, \mathrm{p}<0.002 \mathrm{~b}, \mathrm{c}$ \\
\hline Disease duration at PET (months) [range] & - & $7.9(4.9)[2-24]$ & $9.0(9.3)[1-62]$ & $T_{77}=0.6, p=0.5$ \\
\hline Baseline assessment to PET scan (months) [range] & $43(2.8)$ [39-49] & $1.9(1.2)[0.2-4.7]$ & $2.1(1.3)[0.3-5.9]$ & $\mathrm{T}_{77}=0.6, \mathrm{p}=0.5$ \\
\hline Baseline—repeat assessment (months) [range] & $20.0(1.2)[18-24]$ & $18.5(0.68)[17-20]$ & $18.6(0.67)[17-20]$ & $\mathrm{T}_{77}=0.4, \mathrm{p}=0.7$ \\
\hline LEDD baseline & - & $144(115)$ & $191(140)$ & $\mathrm{T}_{77}=1.6, \mathrm{p}=0.1$ \\
\hline LEDD 18 months & - & $299(154)^{* *}$ & $410(206)^{* *}$ & $\mathrm{~T}_{69}=2.6, \mathrm{p}=0.013$ \\
\hline UPDRS III baseline & - & $23.2(9.0)$ & $28.3(12.6)$ & $\mathrm{T}_{77}=2.2, \mathrm{p}=0.043$ \\
\hline UPDRS III 18 months & - & $33.5(12.0)^{* *}$ & $37.8(10.7)^{* *}$ & $\mathrm{~T}_{69}=1.6, \mathrm{p}=0.1$ \\
\hline MMSE baseline & $29.5(0.7)$ & $29.0(0.9)$ & $27.9(1.5)$ & $F_{2,96}=15, p=0.001 b, c$ \\
\hline MMSE 18 months & $29.6(1.0)$ & $28.9(1.1)$ & $27.1(1.8)^{\star *}$ & $F_{2,88}=23, p=0.001 b, c$ \\
\hline MoCA baseline & $27.7(1.9)$ & $26.3(2.6)$ & $22.4(3.6)$ & $F_{2,89}=27, \mathrm{p}=0.001 \mathrm{~b}, \mathrm{c}$ \\
\hline MoCA 18 months & $27.6(2.4)$ & $26.7(2.6)$ & 23.1 (3.6) & $F_{2,88}=19, \mathrm{p}=0.001 \mathrm{~b}, \mathrm{c}$ \\
\hline
\end{tabular}

Comparisons are either three-group ANOVA, or PD-NC versus PD-MCl unpaired t-test, or (for sex) $\chi^{2}$.

Baseline MoCA scores only available on 72 PD participants. Tukey post hoc tests $p<0.01, a=P D-N C$ versus controls, $b=P D-M C l$ versus controls, $C=P D-M C l$ versus PD-NC.

Longitudinal change paired tests: * $p<0.05$ within group; ${ }^{* *} p<0.01$ within group.

ANOVA, analysis of variance; F, female; LEDD, levodopa equivalent daily dose; M, male; MMSE, Mini-Mental State Examination; MoCA, Montreal Cognitive Assessment; PD-MCI,

Parkinson's disease-mild cognitive impairment; PD-NC, Parkinson's disease-normal cognitive function; PET, positron emission tomography; UPDRS III, motor subsection of Unified

Parkinson's Disease Rating Scale.
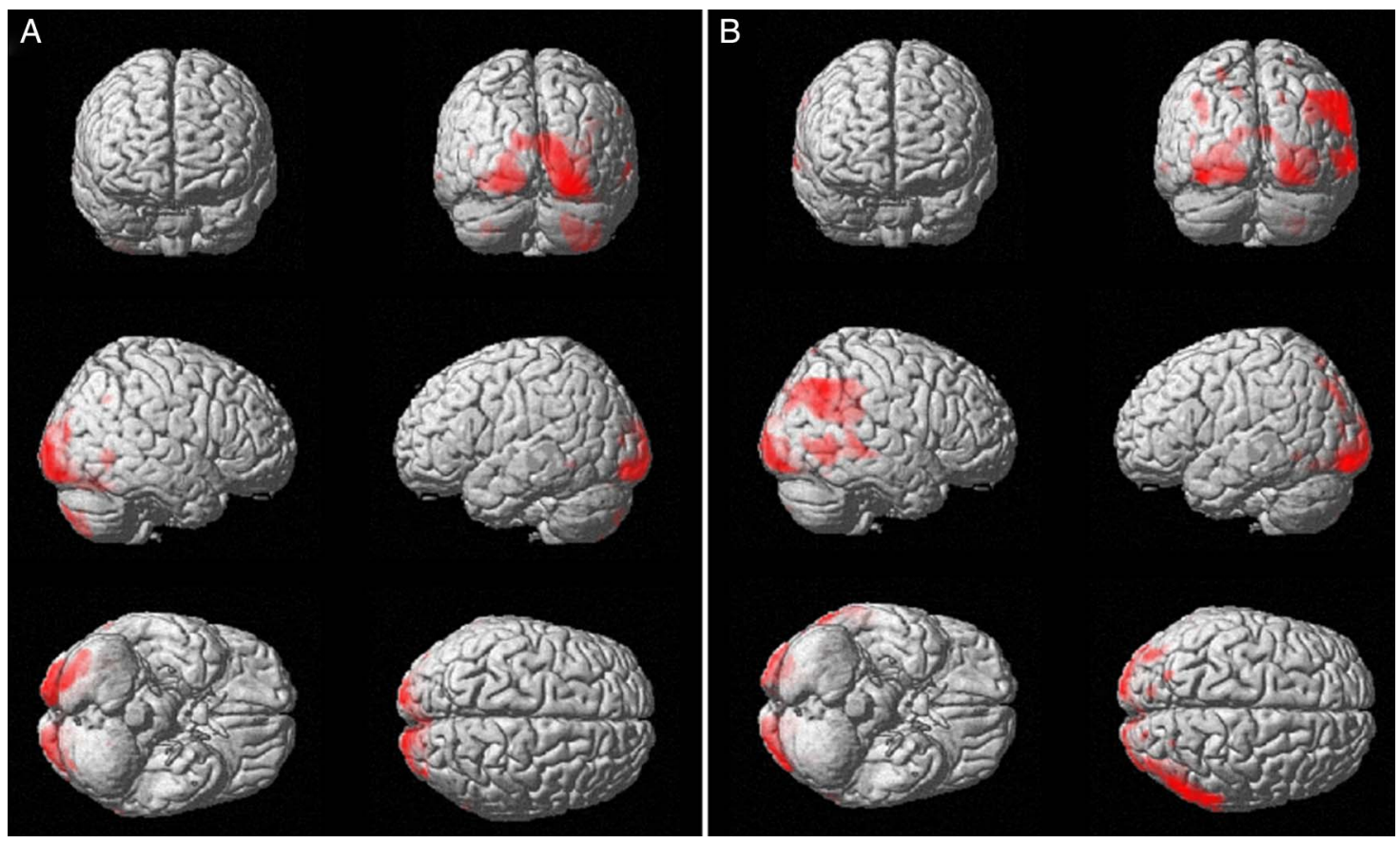

Figure 1 Regions where FDG metabolism is greater in controls than (A) PD-NC and (B) PD-MCI. FDG uptake is normalised to whole brain. SPM $t$ image thresholded at $p<0.05$ FWE corrected for multiple comparisons. FDG, 18F-fluorodeoxyglucose; FWE, family wise error; PD-MCl, Parkinson's disease-mild cognitive impairment; PD-NC, Parkinson's disease-normal cognitive function; SPM, statistical parametric mapping.

differences between PD-NC and PD-MCI in any region (ie, no significant clusters after $\mathrm{p}<0.001$ uncorrected threshold). In both PD-NC and PD-MCI, there was a small bilateral region in the frontal white matter where FDG metabolism was greater than controls (figure 2).

In the PD group as a whole, we performed voxelwise correlations between FDG metabolism and cognitive scores at baseline. Performance on SRM, PAL and OTS correlated with bilateral parietal-temporal FDG metabolism (poor performance associated with reduced metabolism). Furthermore, digit vigilance accuracy correlated with reduced frontal FDG metabolism (figure 3). There were no significant correlations for PRM, pentagons or verbal fluency.

The FDG angular gyrus/cerebellum uptake ratio was significantly different between groups $\left(F_{2,96}, \mathrm{p}<0.001\right)$ with the PD-MCI (ratio=1.05, SD 0.11) being significantly lower (Tukey post hoc $\mathrm{p}<0.01)$ than both PD-NC $(1.12$, SD 0.08) and controls (1.15, SD 0.083) with no significant difference between control and PD-NC. The ratio correlated with MMSE $(r=0.28$; $\mathrm{p}=0.011)$ and MoCA scores $(\mathrm{r}=0.27 ; \mathrm{p}=0.024)$ in the PD 

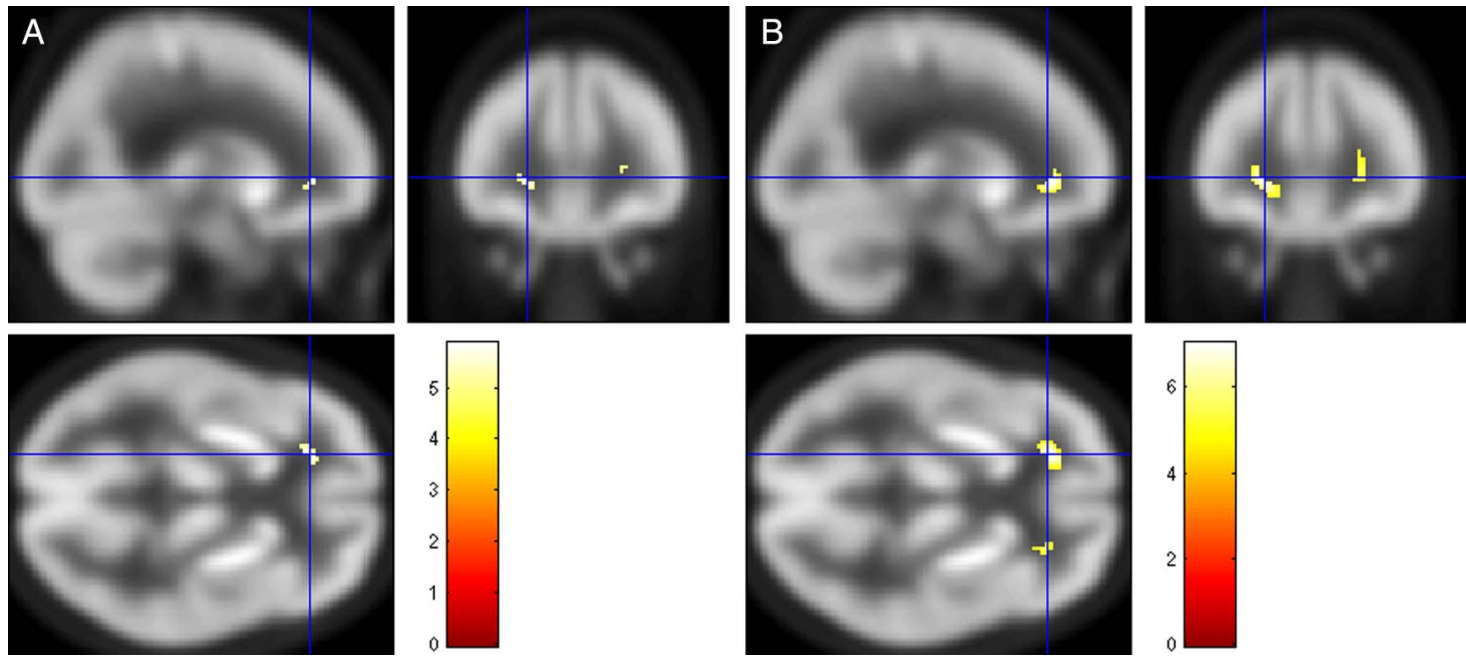

Figure 2 Regions where FDG metabolism is lower in controls than (A) PD-NC and (B) PD-MCl. FDG uptake is normalised to whole brain. SPM $t$ image thresholded at $\mathrm{p}<0.05$ FWE corrected for multiple comparisons. (cross-hair at $-20,36,0)$. FDG, 18F-fluorodeoxyglucose; FWE, family wise error; PD-MCl, Parkinson's disease-mild cognitive impairment; PD-NC, Parkinson's disease-normal cognitive function; SPM, statistical parametric mapping.

participants at baseline. The FDG angular gyrus/cerebellum uptake ratio also significantly predicted both MMSE and MoCA at 18 months, controlling for baseline score, age, sex and years of education. This remained significant even after the addition of disease duration, LEDD, UPDRS III and MCI group at baseline into the model (table 2). To investigate whether FDG uptake in other regions predicted MMSE scores longitudinally, we also performed a voxelwise analysis of MMSE at 18 months with FDG uptake, controlling for baseline MMSE, age, sex and education, and found significant correlations in the lateral parietotemporal-occipital region, but not elsewhere (see online supplementary figure S1).

\section{DISCUSSION}

This is the largest study to date to examine FDG-PET findings in early PD. In this group of newly diagnosed PD, we found that cortical glucose metabolism was reduced posteriorly, particularly in the occipital lobe. The PD-MCI participants demonstrated greater involvement of the parietal and posterior temporal lobes. Notably, FDG metabolism at baseline in the angular gyrus relative to cerebellum predicted MMSE and MoCA scores at 18 months.

Relatively few studies have investigated FDG in early stage PD. Pappatà et $a l^{22}$ studied 24 newly diagnosed, drug naïve patients with PD (half with $\mathrm{MCI}$ ) and found no differences between cognitively normal PD versus controls, while the PD-MCI had parieto-occipital (though not primary visual cortex) and frontal reductions versus controls. The parieto-occipital reductions were not significantly different between PD-MCI and PD-NC. In PD with 3 years of symptom duration, Teune et $a l^{23}$ found metabolic reductions in the occipital pole, inferior parietal and prefrontal cortex, primarily contralateral to the most affected side. In more advanced PD, Garcia-Garcia et $a l^{10}$ demonstrated occipital pole and inferior parietal, and to a lesser extent frontal and temporal FDG reductions in PD-MCI versus controls. The PD-NC participants did not show any metabolic differences relative to controls, and only small differences relative to PD-MCI suggesting that they fell intermediate between control and PD-MCI. Bohnen et al found PD-NC with focal reductions of FDG uptake in the occipital lobe, while participants with PD and dementia had more widespread posterior reductions, and Baba et $a l^{24}$ found that PD with severe olfactory dysfunction were both more likely to develop dementia, and also had more pronounced parieto-occipitotemporal FDG reductions.

Although our parieto-occipital changes are broadly in agreement with these studies, we saw fewer frontal FDG reductions in our PD-MCI group. This may in part be due to differences in the cognitive tests used to define MCI. This varies between studies both in terms of which particular tests are chosen, but also the number per cognitive domain (the MDS guidelines ${ }^{17}$ suggest a minimum of two tests per domain, but no maximum). We had relatively limited assessment of visual and language function, and our choice of memory tasks were largely dependent on visual cues, which may have resulted in more posterior metabolic deficits being identified. Our participants were scanned and cognitively assessed while taking their usual dopaminergic medication, and this may have influenced the scores and FDG uptake.

Eidelberg and colleagues have used principal component analysis to identify network patterns in PD. They have found two consistent patterns: (1) a PD-related covariance pattern (PDRP) of relative increase in the basal ganglia, thalamus and cerebellum, and decrease in premotor and parieto-occipital cortex, and (2) a PD-related cognitive pattern of reduced FDG in precuneus, inferior parietal/angular gyrus, pre supplementary motor area (preSMA), with increases in the cerebellum, the expression of which increases in patients with poorer cognitive function. ${ }^{4} 25$

In our study, parietal hypometabolism predicted future cognitive function as measured by MMSE and MoCA at 18 months controlling for the baseline score. This is in keeping with smaller studies in more advanced $\mathrm{PD}^{6-8}$ where FDG reductions in parieto-occipital and temporal regions have been associated with cognitive decline, or progression to dementia. The inferior parietal lobes and precuneus region are strongly affected in Alzheimer's disease and DLB. ${ }^{9}{ }^{23}$ In AD, glucose hypometabolism is a relatively early feature, preceding cortical atrophy, and predicting cognitive decline at an early stage. ${ }^{11}$ In $\mathrm{PD}$, this region shows reduced FDG and blood flow in the absence of atrophy, ${ }^{56}$ suggesting that in PD also, reduced metabolism is a relatively early feature, possibly reflecting synaptic dysfunction due to local $\alpha$-synuclein pathology, ${ }^{27} 28$ which precedes atrophy and cognitive decline. 

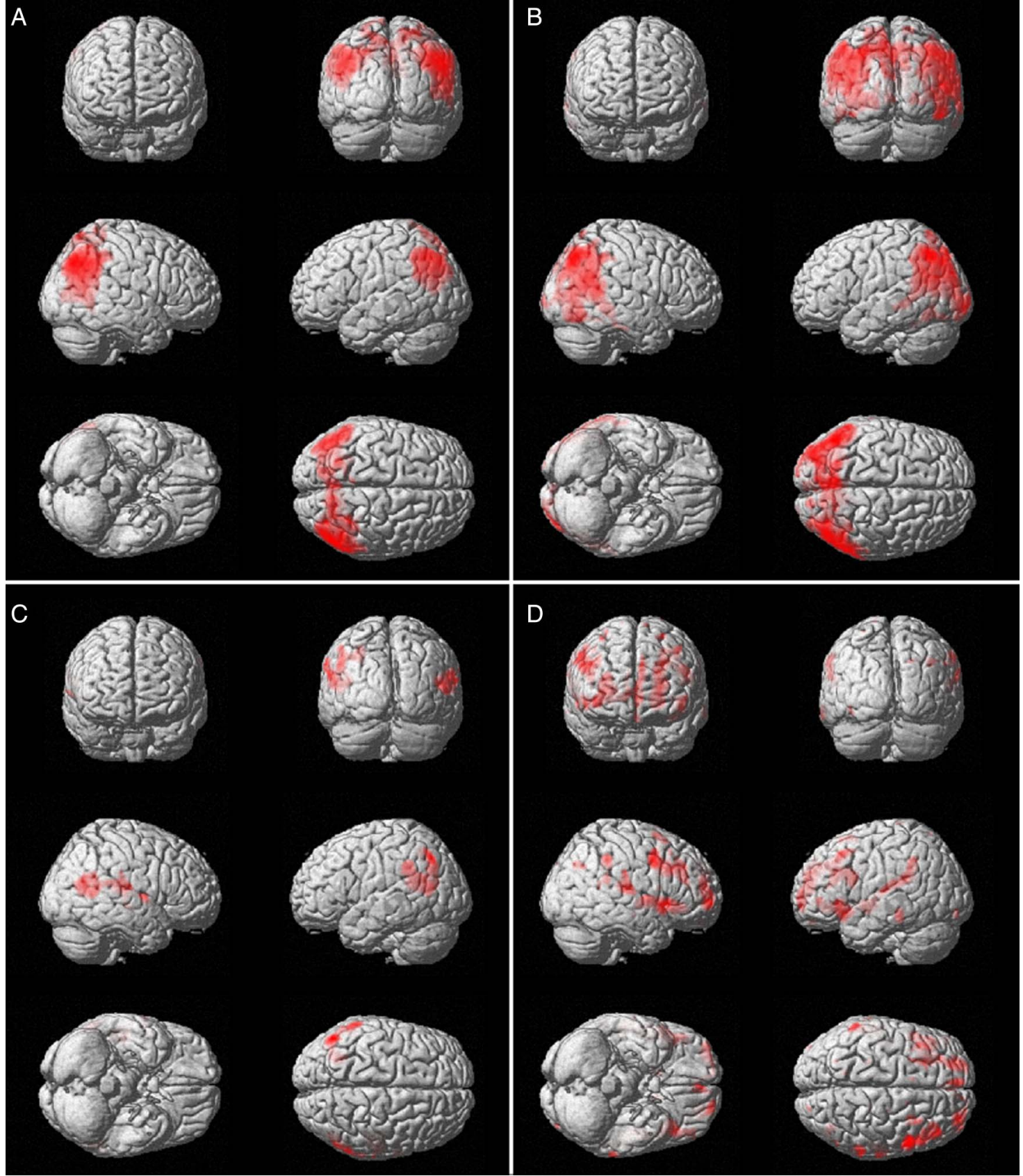

Figure 3 Regions where FDG metabolism correlates with cognitive scores. (A) Spatial Recognition Memory, (B) Paired Associates Learning; (C) One Touch Stockings; (D) Digit Vigilance. In all, higher FDG is associated with better performance. SPM $t$ image voxelwise thresholded at $p<0.001$ uncorrected for multiple comparisons, significant clusters at $\mathrm{p}<0.05$ FWE corrected for multiple comparisons. FDG, 18F-fluorodeoxyglucose; FWE, family wise error; SPM, statistical parametric mapping.

We found prominent reduction in FDG of the occipital pole in all PD participants, and an association was demonstrated between occipital FDG and performance on PAL (which requires discrimination of different shapes, and retention of their location). A number of other studies have also found occipital FDG reductions in $\mathrm{PD},{ }^{5} 810$ and relatively reduced occipital FDG is a component of the PDRP. ${ }^{29}$ Previous research has found associations of occipital reduction with visuospatial deficits. ${ }^{10} 30$ However, the occipital pole hypometabolism is similar in PD with dementia to cognitively normal PD, ${ }^{10} 31$ and does not seem related to the severity of motor features. $^{32} 33$ Occipital hypoperfusion/metabolism is also a distinctive feature of $\mathrm{DLB},{ }^{34}$ in which it does not vary with severity of parkinsonism. ${ }^{35}$ There may be an association with cholinergic function, since Shimada et $a l^{36}$ established prominent occipital loss of AChE activity (with $\mathrm{N}$-(11C)-methyl-4piperidyl acetate) in PD at all stages, and Fong et $a l^{37}$ found (in a small group of DLB) that acute administration of a cholinesterase inhibitor was associated with increased occipitoparietal perfusion.

Reduced occipital FDG uptake has been associated with rapid eye movement (REM) sleep behaviour disorder (RBD), ${ }^{38} 39$ a clinical feature that may precede or accompany DLB. It may be that patients with more profound occipital FDG reductions are at risk of developing clinical features of DLB such as RBD or visual hallucinations. 
Table 2 Predictors of MMSE and MoCA at 18 months

\begin{tabular}{|c|c|c|c|c|}
\hline & $\begin{array}{l}\text { MMSE at } 18 \text { months } \\
\text { Model } 1(R=0.564, \\
\text { adj- } R^{2}=0.265 . \text { Adj- } R^{2} \text { was } \\
\text { increased by } 0.124 \text { with FDG) }\end{array}$ & $\begin{array}{l}\text { MMSE at } 18 \text { months } \\
\text { Model } 2(R=0.642 \\
\text { adj- } R^{2}=0.325 . \text { Adj- } R^{2} \text { was } \\
\text { increased by } 0.055 \text { with FDG) }\end{array}$ & $\begin{array}{l}\text { MoCA at } 18 \text { months } \\
\text { Model } 1(R=0.767 \\
\text { adj- } R^{2}=0.553 . \text { Adj- } R^{2} \text { was } \\
\text { increased by } 0.071 \text { with FDG) }\end{array}$ & $\begin{array}{l}\text { MoCA at } 18 \text { months } \\
\text { Model } 2(R=0.798 \\
\text { adj- } R^{2}=0.578 . \text { Adj- } R^{2} \text { was } \\
\text { increased by } 0.065 \text { with FDG) }\end{array}$ \\
\hline MMSE baseline & $0.33(0.11) p=0.005$ & $0.22(0.11) p=0.057$ & - & - \\
\hline MoCA baseline & - & - & $0.65(0.09) p<0.001$ & $0.61(0.10) p<0.001$ \\
\hline FDG parietal/cerebellum & $0.38(0.11) p=0.001$ & $0.28(0.11) p=0.016$ & $0.30(0.09) p=0.002$ & $0.29(0.09) p=0.003$ \\
\hline Age at baseline & $-0.05(0.11) p=0.7$ & $0.02(0.11) p=0.9$ & $-0.07(0.09) p=0.5$ & $-0.09(0.09) p=0.4$ \\
\hline Female & $0.02(0.11) p=0.9$ & $0.06(0.11) p=0.6$ & $0.03(0.09) \mathrm{p}=0.8$ & $-0.01(0.09) p=0.9$ \\
\hline Years education & $0.04(0.10) p=0.7$ & $-0.08(0.11) p=0.9$ & $-0.14(0.09) \mathrm{p}=0.1$ & $-0.14(0.09) p=0.12$ \\
\hline $\begin{array}{l}\text { Disease duration } \\
\text { baseline }\end{array}$ & - & $-0.02(0.10) p=0.9$ & - & $0.00(0.10) p=0.9$ \\
\hline LEDD baseline & - & $-0.09(0.09) p=0.4$ & - & $0.18(0.09) p=0.048$ \\
\hline UPDRS III baseline & - & $-0.05(0.10) p=0.6$ & - & $0.11(0.09) \mathrm{p}=0.2$ \\
\hline PD-MCI baseline & - & $-0.33(0.12) p=0.007$ & - & $-0.17(0.11) p=0.13$ \\
\hline
\end{tabular}

Unlike most studies, we acquired FDG-PET imaging with eyes open. Bohnen et $a l^{8}$ also acquired their data in patients with eyes open, and they found prominent primary visual deficits in PD-NC. Using MRI arterial spin labelling in PD-NC with eyes open, Fernández-Seara et $\mathrm{al}^{40}$ also observed prominent occipital deficits. Conversely, some of the FDG studies with eyes closed $^{622}$ did not find occipital pole reductions in PD. Occipital glucose metabolism is generally greater with eyes open. ${ }^{41}$ It is possible that patients with PD engage less with the visual environment due to changes in the retina, ${ }^{42}$ or cholinergic loss. ${ }^{43}$ In this case, the increase in glucose metabolism on eyes opening would be less marked in PD, and hence any occipital hypometabolism made more obvious compared with control participants. Further research is required to investigate the origin and implications of this occipital pole metabolic reduction.

Strengths of our study include the large, well-characterised cohort at the earliest stages of PD. Weaknesses include the relatively limited testing of visuospatial function. Although we saw a significant correlation between parietal FDG and decline in MMSE and MoCA, the association was not strong enough to allow FDG to be used for prediction on an individual basis. This may partly be due to the limited degree of cognitive decline in an early PD group over a relatively short period of follow-up. Also, quantifying change using repeated cognitive assessment is difficult due to practice effects, and influence of factors such as motivation on the scores. With longer follow-up, the value of baseline FDG scanning and other biomarkers to predict cognitive decline should become more evident.

In conclusion, we found occipital reductions in a large cohort of newly diagnosed patients with PD relative to age-matched controls. Reductions in the temporoparietal region were associated with poorer cognitive performance. The FDG parietal/ cerebellar ratio shows some promise for predicting global cognitive decline in early PD.

\footnotetext{
Author affiliations

${ }^{1}$ Institute of Neuroscience and Newcastle University Institute for Ageing, Newcastle University, Newcastle upon Tyne, UK

${ }^{2}$ Department of Geriatric Medicine, University of Edinburgh, Edinburgh, UK

${ }^{3}$ School of Medicine \& Menzies Health Institute Queensland, Griffith University, Gold Coast, Queensland, Australia

${ }^{4}$ Department of Nuclear Medicine, Freeman Hospital, Newcastle upon Tyne, UK

${ }^{5}$ Department of Psychiatry, University of Cambridge, Cambridge, UK
}

${ }^{6}$ John van Geest Centre for Brain Repair, University of Cambridge, Cambridge, UK ${ }^{7}$ Northern Institute for Cancer Research, Newcastle University, Newcastle upon Tyne, UK

${ }^{8}$ Division of Neuroscience, Imperial College London, London, UK

${ }^{9}$ Institute of Clinical Medicine, Aarhus University, Denmark

Acknowledgements The authors wish to thank all participants and their carers for their valuable time engaging with this research.

Contributors MJF performed the data analysis, and drafted the paper. AJY, GWD, TKK, and RAL were involved in data collection and participant recruitment, assisted with the interpretation of the results, and provided comments and additional suggestions for revisions of the draft. RJM designed the imaging protocol, and critically reviewed the manuscript. GSP was involved in data analysis design and critically reviewed the manuscript. DJBu, RAB, DJBr and JTO obtained funding for the project, designed the imaging protocol, assisted with recruitment of study participants, assisted with the interpretation of the results and reviewed the manuscript. All authors approved the final manuscript.

Funding The authors acknowledge the study funders, Parkinson's UK (grant code J-0802), Lockhart Parkinson's Disease Research Fund and Michael J Fox Foundation (MJFF). The research was supported by the NIHR Newcastle Biomedical Research Unit based at Newcastle upon Tyne Hospitals NHS Foundation Trust/Newcastle University, and an NIHR Biomedical Research Centre award to the University of Cambridge/Addenbrooke's Hospital. Newcastle University and the University of Cambridge acknowledge the support of the NIHR, through the Dementias and Neurodegenerative Diseases Research Network.

Disclaimer The views expressed are those of the authors and not necessarily those of the NHS, the NIHR, or the Department of Health.

Competing interests GSP has received honoraria from GE Healthcare. AJY has received honoraria from Teva-Lundbeck and sponsorship from Teva-Lundbeck, UCB, GSK, Genus, Britannia and Abbvie for attending conferences. DJBu reports grants from Parkinson's UK, NIHR, Wellcome Trust and the Michael J Fox Foundation. RAB has grants from NIHR, EU, Parkinson's UK, CPT, Rosetrees Trust. JTO reports grants from the Medical Research Council, NIHR, ARUK and the Alzheimer's Society and has acted as a consultant for GE Healthcare, Lilly, TauRx, Cytox and Axon.

Ethics approval The Newcastle and North Tyneside Research Ethics Committee.

Provenance and peer review Not commissioned; externally peer reviewed.

Open Access This is an Open Access article distributed in accordance with the terms of the Creative Commons Attribution (CC BY 4.0) license, which permits others to distribute, remix, adapt and build upon this work, for commercial use, provided the original work is properly cited. See: http://creativecommons.org/licenses/ by/4.0/

\section{REFERENCES}

1 Dorsey ER, Constantinescu R, Thompson JP, et al. Projected number of people with Parkinson diseases in the most populous nations, 2005 through 2030. Neurology 2007;68:384-6. 
2 Yarnall AJ, Breen DP, Duncan GW, et al. Characterising mild cognitive impairment in incident Parkinson disease: the ICICLE-PD study. Neurology 2014;82:308-16.

3 Aarsland D, Andersen K, Larsen JP, et al. Prevalence and characteristics of dementia in Parkinson disease —an 8-year prospective study. Arch Neurol 2003;60:387-92.

4 Meles SK, Tang CC, Teune LK, et al. Abnormal metabolic pattern associated with cognitive impairment in Parkinson's disease: a validation study. J Cereb Blood Flow Metab 2015;35:1478-84.

5 González-Redondo R, García-García D, Clavero P, et al. Grey matter hypometabolism and atrophy in Parkinson's disease with cognitive impairment: a two-step process. Brain 2014;137:2356-67.

6 Shoji Y, Nishio Y, Baba T, et al. Neural substrates of cognitive subtypes in Parkinson's disease: a 3-year longitudinal study. PLoS ONE 2014;9:e110547.

7 Tard C, Demailly F, Delval A, et al. Hypometabolism in posterior and temporal areas of the brain is associated with cognitive decline in Parkinson's disease. J Parkinsons Dis 2015:5:569-74.

8 Bohnen NI, Koeppe RA, Minoshima S, et al. Cerebral glucose metabolic features of Parkinson disease and incident dementia: longitudinal study. J Nucl Med 2011;52:848-55.

9 O'Brien JT, Firbank MJ, Davison C, et al. FDG-PET and perfusion SPECT in the diagnosis of Alzheimer's disease and Lewy body dementias. J Nucl Med 2014;55:1959-65.

10 Garcia-Garcia D, Clavero P, Gasca Salas C, et al. Posterior parietooccipital hypometabolism may differentiate mild cognitive impairment from dementia in Parkinson's disease. Eur J Nucl Med Mol Imaging 2012;39:1767-77.

11 Jack CR, Knopman DS, Jagust WJ, et al. Hypothetical model of dynamic biomarkers of the Alzheimer's pathological cascade. Lancet Neurol 2010;9:119-28.

12 Hughes AJ, Daniel SE, Kilford L, et al. Accuracy of clinical diagnosis of idiopathic Parkinson's disease: a clinico-pathological study of 100 cases. J Neurol Neurosurg Psychiatr 1992; 55:181-4.

13 Goetz CG, Tilley BC, Shaftman SR, et al. Movement Disorder Society sponsored revision of the Unified Parkinson's Disease Rating Scale (MDS-UPDRS): scale presentation and clinimetric testing results. Mov Disord 2008;23:2129-70.

14 Hoehn MM, Yahr MD. Parkinsonism: onset, progression and mortality. Neurology 1967; 17:427-42.

15 Folstein MF, Folstein SE, McHugh PR. A practical method for grading the cognitive state of patients for the clinician. J Psychiatr Res 1975;12:189-98.

16 Dalrymple-Alford JC, MacAskill MR, Nakas CT, et al. The MoCA: well-suited screen for cognitive impairment in Parkinson disease. Neurology 2010;75:1717-25.

17 Litvan I, Goldman JG, Tröster Al, et al. Diagnostic criteria for mild cognitive impairment in Parkinson's disease: Movement Disorder Society Task Force guidelines. Mov Disord 2012;27:349-56.

18 Wesnes KA, McKeith IG, Ferrara $R$, et al. Effects of rivastigmine on cognitive function in dementia with Lewy bodies: a randomised placebo-controlled international study using the cognitive drug research computerised assessment system. Dement Geriatr Cogn Disord 2002;13:183-92.

19 Fray PJ, Robbins TW. CANTAB battery: proposed utility in neurotoxicology. Neurotoxicol Teratol 1996;18:499-504.

20 Tomlinson CL, Stowe R, Patel S, et al. Systematic review of levodopa dose equivalency reporting in Parkinson's disease. Mov Disord 2010;25:2649-53.

21 Della Rosa PA, Cerami C, Gallivanone F, et al. A standardized [18F]-FDG-PET template for spatial normalization in statistical parametric mapping of dementia. Neuroinformatics 2014;12:575-93.

22 Pappatà S, Santangelo G, Aarsland D, et al. Mild cognitive impairment in drug-naive patients with PD is associated with cerebral hypometabolism. Neurology 2011:77:1357-62.
23 Teune LK, Bartels AL, de Jong BM, et al. Typical cerebral metabolic patterns in neurodegenerative brain diseases. Mov Disord 2010;25:2395-404.

24 Baba T, Kikuchi A, Hirayama K, et al. Severe olfactory dysfunction is a prodromal symptom of dementia associated with Parkinson's disease: a 3 year longitudinal study. Brain 2012;135(Pt 1):161-9.

25 Huang $C$, Mattis $P$, Tang $C$, et al. Metabolic brain networks associated with cognitive function in Parkinson's disease. Neurolmage 2007;34:714-23.

26 Firbank MJ, Colloby SJ, Burn DJ, et al. Regional cerebral blood flow in Parkinson's disease with and without dementia. Neuroimage 2003;20:1309-19.

27 Jellinger KA. The role of $\alpha$-synuclein in neurodegeneration-an update. Trans/ Neurosci 2012;3:75-122.

28 Braak H, Ghebremedhin E, Rüb U, et al. Stages in the development of Parkinson's disease-related pathology. Cell Tissue Res 2004;318:121-34. http://dx.doi.org/ 10.1007/s00441-004-095 6-9

29 Ma Y, Tang C, Spetsieris PG, et al. Abnormal metabolic network activity in Parkinson's disease: test-retest reproducibility. J Cereb Blood Flow Metab 2007;27:597-605.

30 Uchiyama M, Nishio Y, Yokoi K, et al. Pareidolia in Parkinson's disease without dementia: a positron emission tomography study. Parkinsonism Relat Disord 2015;21:603-9.

31 Hosokai Y, Nishio Y, Hirayama K, et al. Distinct patterns of regional cerebral glucose metabolism in Parkinson's disease with and without mild cognitive impairment. Mov Disord 2009;24:854-62.

32 Huang C, Tang C, Feigin A, et al. Changes in network activity with the progression of Parkinson's disease. Brain 2007;130:1834-46.

33 Berti V, Polito C, Ramat S, et al. Brain metabolic correlates of dopaminergic degeneration in de novo idiopathic Parkinson's disease. Eur J Nucl Med Mol Imaging 2010:37:537-44.

34 Mak E, Su L, Williams GB, et al. Neuroimaging characteristics of dementia with Lewy bodies. Alzheimers Res Ther 2014;6:18.

35 Takahashi R, Ishii K, Shimada K, et al. Hypoperfusion of the motor cortex associated with parkinsonism in dementia with Lewy bodies. J Neuro/ SCi 2010:288:88-91.

36 Shimada $\mathrm{H}$, Hirano $\mathrm{S}$, Shinotoh $\mathrm{H}$, et al. Mapping of brain acetylcholinesterase alterations in Lewy body disease by PET. Neurology 2009;73:273-8.

37 Fong TG, Inouye SK, Dai W, et al. Association cortex hypoperfusion in mild dementia with Lewy bodies: a potential indicator of cholinergic dysfunction. Brain Imaging Behav 2011;5:25-35.

38 Fujishiro $\mathrm{H}$, Iseki $\mathrm{E}$, Kasanuki K, et al. Glucose hypometabolism in primary visual cortex is commonly associated with clinical features of dementia with Lewy bodies regardless of cognitive conditions. Int J Geriatr Psychiatry 2012;27: 1138-46.

39 Ge JJ, Wu P, Peng SC, et al. Assessing cerebral glucose metabolism in patients with idiopathic rapid eye movement sleep behavior disorder. J Cereb Blood Flow Metab 2015;35:2062-9

40 Fernández-Seara MA, Mengual E, Vidorreta M, et al. Cortical hypoperfusion in Parkinson's disease assessed using arterial spin labeled perfusion MRI. Neurolmage 2012:59:2743-50.

41 Riedl V, Bienkowska K, Strobel C, et al. Local activity determines functional connectivity in the resting human brain: a simultaneous FDG-PET/fMRI study. J Neurosci 2014;34:6260-6.

42 Archibald NK, Clarke MP, Mosimann UP, et al. The retina in Parkinson's disease. Brain 2009:132:1128-45.

43 Yarnall AJ, Rochester L, Burn DJ. The interplay of cholinergic function, attention, and falls in Parkinson's disease. Mov Disord 2011:26:2496-503. 